\title{
Exploring optimal topology of thermal cloaks by CMA-ES
}

Cite as: Appl. Phys. Lett. 112, 061108 (2018); https://doi.org/10.1063/1.5016090

Submitted: 16 November 2017 • Accepted: 20 January 2018 • Published Online: 09 February 2018

Garuda Fujii, (D) Youhei Akimoto and Masayuki Takahashi

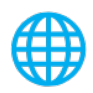

View Online

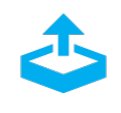

Export Citation

\section{ARTICLES YOU MAY BE INTERESTED IN}

Optimizing the structural topology of bifunctional invisible cloak manipulating heat flux and direct current

Applied Physics Letters 115, 174101 (2019); https://doi.org/10.1063/1.5123908

Shaped graded materials with an apparent negative thermal conductivity

Applied Physics Letters 92, 251907 (2008); https://doi.org/10.1063/1.2951600

Direct-current electric invisibility through topology optimization

Journal of Applied Physics 123, 233102 (2018); https://doi.org/10.1063/1.5022881

\section{Challenge us.}

What are your needs for periodic signal detection?

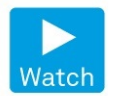

Zurich

- Instruments

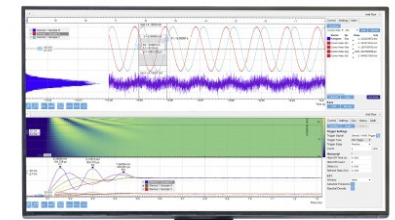

? 


\title{
Exploring optimal topology of thermal cloaks by CMA-ES
}

\author{
Garuda Fujii, ${ }^{1, a)}$ Youhei Akimoto, ${ }^{1}$ and Masayuki Takahashi ${ }^{2}$ \\ ${ }^{1}$ Institute of Engineering, Shinshu University, Nagano 380-8553, Japan \\ ${ }^{2}$ Graduate School of Science and Technology, Shinshu University, Nagano 380-8553, Japan
}

(Received 16 November 2017; accepted 20 January 2018; published online 9 February 2018)

\begin{abstract}
This paper presents topology optimization for thermal cloaks expressed by level-set functions and explored using the covariance matrix adaptation evolution strategy (CMA-ES). Designed optimal configurations provide superior performances in thermal cloaks for the steady-state thermal conduction and succeed in realizing thermal invisibility, despite the structures being simply composed of iron and aluminum and without inhomogeneities caused by employing metamaterials. To design thermal cloaks, a prescribed objective function is used to evaluate the difference between the temperature field controlled by a thermal cloak and when no thermal insulator is present. The CMA-ES involves searches for optimal sets of level-set functions as design variables that minimize a regularized fitness involving a perimeter constraint. Through topology optimization subject to structural symmetries about four axes, we obtain a concept design of a thermal cloak that functions in an isotropic heat flux. Published by AIP Publishing. https://doi.org/10.1063/1.5016090
\end{abstract}

Optical invisibility has been actively pursued over the past decade ever since cloaking devices were proposed, ${ }^{1,2}$ and metamaterials, ${ }^{3}$ metasurfaces, ${ }^{4}$ and scattering cancellation ${ }^{5}$ were developed to realize optical invisibility. The concept of optical invisibility has spread by analogy to not only far-infrared cloaks $^{6}$ and external cloaks ${ }^{7}$ but also acoustic cloaks, ${ }^{8,9}$ direct current (DC) electric cloaks, ${ }^{10,11}$ and fluid flow cloaks. ${ }^{12}$

Topology optimization ${ }^{13}$ has recently been applied to the design of, for example, optical cloaks, ${ }^{14-18}$ carpet cloaks, ${ }^{18,19}$ and DC electric cloaks. ${ }^{20}$ It admits not only changes in the shape but also the creation of new holes during optimization and has a higher degree of design freedom than sizing and shape optimizations. Indeed, cloaking structures designed through topology optimizations provide superior performances despite their simple composition using bulk isotropic materials.

By analogy, thermal cloaks ${ }^{21,22}$ are being developed to eliminate disturbances caused by obstacles from heat flows. These cloaks are composed of thermal metamaterials configured based on transformation thermodynamics. ${ }^{23-25}$ Microstructures of thermal metamaterials made of composite materials are also designed using optimizations. ${ }^{26}$ However, these approaches require resolving challenging configuration problems because the metamaterial microstructures are complicated. To overcome these drawbacks, a bilayer thermal cloak made of bulk isotropic materials ${ }^{27,28}$ and an ultrathin thermal cloak in three dimensions ${ }^{29}$ are proposed. The devices can be developed without inhomogeneity; specifically, they can be made using only naturally available materials, which simplifies their fabrication.

In this work, we present sophisticated designs of thermal cloaks composed of bulk isotropic materials using the covariance matrix adaptation evolution strategy (CMA-ES) to explore the optimal topology of structures expressed as a level-set function. CMA-ES is known as a powerful stochastic method for difficult non-linear optimization problems, namely, multimodal, non-separable, and non-continuous

a)g_fujii@shinshu-u.ac.jp optimization problems. A level set method clearly expresses structural boundaries using the isosurfaces of the level set functions, which become positive within a structure and negative outside of the structure. Thermal cloaks are designed to mimic the thermal distribution that emerges when no insulating obstacles exist and retains the external conductive heat flux.

The scheme of this topology optimization for thermal cloaks is illustrated in Fig. 1(a). The thermal insulating obstacle is denoted by $\Omega_{\text {ins }}$, and the fixed design domain $\Omega_{\mathrm{D}}$ is set to cover the insulator. The structure of a thermal cloak $\Omega_{\mathrm{S}}$ is designed in $\Omega_{\mathrm{D}}$. Structural symmetries are imposed about the $x$ and $y$ axes, and the structures are expressed using level-set functions discretized over the grid points in $\Omega_{\mathrm{D}}$ [Fig. 1(b)]. The exterior of the fixed design domain is denoted by $\Omega_{\text {out }}$ in which the disturbance of the temperature distribution is evaluated from the performance of the thermal cloak. Temperatures on the left-most and right-most boundaries of $\Omega_{\text {out }}$ are fixed at $T=T_{\text {low }}$ and $T=T_{\text {high }}$, respectively. The upper and lower boundaries of $\Omega_{\text {out }}$ are thermally insulated.

By controlling the temperature around an obstacle, thermal cloaks are realized by reproducing temperature fields

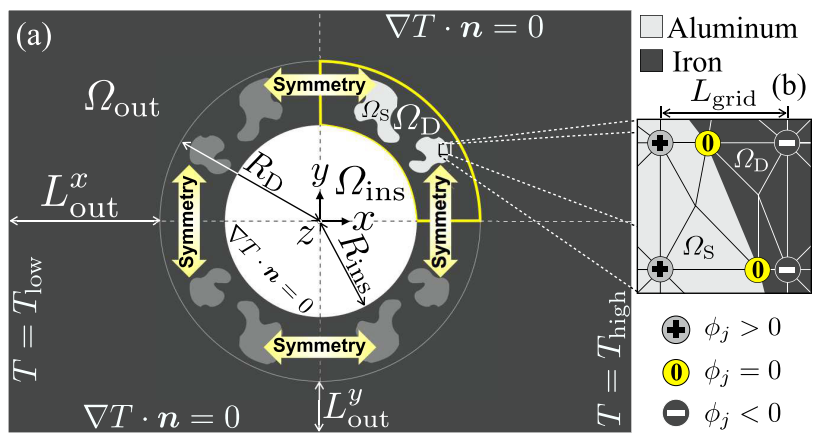

FIG. 1. (a) Schematic of topology optimization for thermal cloaks. Domain sizes are $R_{\text {ins }}=R_{\mathrm{D}} \times 3 / 5, L_{\text {out }}^{x}=R_{\mathrm{D}}, L_{\text {out }}^{y}=R_{\mathrm{D}} \times 1 / 3$, and $L_{\text {grid }}=R_{\mathrm{D}} \times 1 /$ 90. The thermal conductivities of iron and aluminum are set to $\lambda_{\text {iron }}=67$ $(\mathrm{W} / \mathrm{mK})$ and $\lambda_{\text {alum }}=204(\mathrm{~W} / \mathrm{mK})$. (b) Discretized level set functions $\phi_{j}$. 
corresponding to when no insulating obstacle is present. Then, to evaluate the performance of the thermal cloak, the objective function $\Psi$ is set to the least squares error relative to a referenced temperature field, $T_{\text {ref }}$, when no insulating obstacles are present, that is,

$$
\Psi=\frac{1}{\Psi_{\mathrm{n}}} \int_{\Omega_{\mathrm{out}}}\left|T-T_{\mathrm{ref}}\right|^{2} d \Omega,
$$

where $T$ represents the temperature controlled by a thermal cloak. As $\Psi$ is minimized, $T$ tends to $T_{\text {ref }}$ at which the thermal cloak mimics the temperature field when no insulating obstacles are present. The above objective function is normalized by $\Psi_{\mathrm{n}}$ defined as

$$
\Psi_{\mathrm{n}}=\int_{\Omega_{\mathrm{out}}}\left|T_{\text {bare }}-T_{\mathrm{ref}}\right|^{2} d \Omega,
$$

where $T_{\text {bare }}$ represents the temperature when a bare insulating obstacle exists in the absence of cloaking. The temperature fields $T_{\text {ref }}$ and $T_{\text {bare }}$ depend on conditions during steady-state thermal conduction, specifically, the direction of thermal flux, sizes of the cloaked domain, and thermal properties of materials.

As a perimeter constraint, we minimize the perimeter of the structure using the objective function to regularize the topology optimization problem. Then, during optimization, we minimize a fitness function defined as the sum of the objective function $\Psi$ and a scaled value of the perimeter $L_{\mathrm{p}}$

$$
\inf _{\phi} F_{\text {top }}=\Psi+\tau L_{\mathrm{p}}
$$

where $\tau$ represents a regularization parameter. The perimeter $L_{\mathrm{p}}$ normalized by $R_{\mathrm{D}}$ is computed as the boundary length between $\Omega_{\mathrm{S}}$ and $\Omega_{\mathrm{D}} \backslash \Omega_{\mathrm{S}}$. By minimizing the perimeter with $\Psi$, the surface of designed configurations is tightened. A compactness property preventing non-fabricable fine structures from remaining is given during the topology optimization. The strength of the perimeter constraint can be adjusted by changing $\tau$ that determines the ratio between $\Psi$ and $L_{\mathrm{p}}$.

The temperature $T$ obeys the Laplace equation in the steady-state thermal conduction as

$$
\nabla \cdot(\lambda(\boldsymbol{x}) \nabla T)=0
$$

where $\lambda(\boldsymbol{x})$ represents the position-dependent thermal conductivity given by

$$
\lambda(\boldsymbol{x})= \begin{cases}\lambda_{\text {iron }}+\chi\left(\lambda_{\text {alum }}-\lambda_{\text {iron }}\right) & x \in \Omega_{\mathrm{D}} \\ \lambda_{\text {iron }} & x \in \Omega_{\text {out }},\end{cases}
$$

where $\lambda_{\text {iron }}$ and $\lambda_{\text {alum }}$ denote the thermal conductivities of iron and aluminum, respectively. The characteristic function $\chi$ is defined in the fixed design domain as

$$
\chi(\phi(x))=\left\{\begin{array}{lll}
1 & \text { if } & x \in \Omega_{\mathrm{S}} \\
0 & \text { if } & x \in \Omega_{\mathrm{D}} \backslash \Omega_{\mathrm{S}}
\end{array}\right.
$$

where $\phi(\boldsymbol{x})$ represents the level set functions describing the structural boundaries via the isosurfaces. The function becomes positive in designed structure $\Omega_{\mathrm{S}}$, negative outside structures $\Omega_{\mathrm{D}} \backslash \Omega_{\mathrm{S}}$, and zero on structural boundaries $\Gamma_{\mathrm{S}}$, that is,

$$
\begin{cases}-1 \leq \phi(x)<0 & \forall \boldsymbol{x} \in \Omega_{\mathrm{D}} \backslash \Omega_{\mathrm{S}} \\ \phi(x)=0 & \forall \boldsymbol{x} \in \Gamma_{\mathrm{S}} \\ 0<\phi(\boldsymbol{x}) \leq 1 & \forall \boldsymbol{x} \in \Omega_{\mathrm{S}} \backslash \Gamma_{\mathrm{S}}\end{cases}
$$

The level-set functions are discretized to $\phi(\boldsymbol{x})=\sum_{j} s_{j} \phi_{j}$, where $s_{j}$ is the basis function for the linear interpolation and $\phi_{j}$ represents the discretized level-set functions defined on the grid points, see Fig. 1(b). The sets of discretized level-set functions are denoted as $\boldsymbol{\phi}=\left\{\phi_{1}, \phi_{2}, \ldots, \phi_{j}, \ldots, \phi_{N}\right\}$. The vector $\boldsymbol{\phi}$ is optimized using the CMA-ES. To implement the box constraint $-1 \leq \phi(\boldsymbol{x}) \leq 1$, an adaptive penalty function $^{18}$ is employed.

The algorithm adopted for the CMA-ES (Fig. 2) is based on a multiple-point search that adapts the shape, size, and center of the mutation distribution by generating random numbers that follow a normal distribution. With the first generation $g=0$, the parameters of the distributions and the evolution paths are initialized as

$$
\begin{aligned}
& \boldsymbol{m}^{(0)}=\frac{b_{\mathrm{u}}+b_{\mathrm{l}}}{2} \mathbf{1}_{N}, \quad \sigma^{(0)}=0.3\left(b_{\mathrm{u}}-b_{\mathrm{l}}\right), \\
& \boldsymbol{C}^{(0)}=\boldsymbol{I}_{N}, \quad \boldsymbol{p}_{\sigma}^{(0)}=\mathbf{0}, \quad \boldsymbol{p}_{\boldsymbol{c}}^{(0)}=\mathbf{0},
\end{aligned}
$$

where $\boldsymbol{m}^{(g)}$ represents the mean vector, which determines the center of the mutation distribution, $\mathbf{1}_{N}$ is the $n$-dimensional vector with all elements set to $1, \sigma^{(g)}$ the step size representing the spread of the mutation distribution, and $\boldsymbol{I}_{N}$ the identity matrix of dimension $N$. The lower and upper bounds of the design variables are set to $b_{1}=-1$ and $b_{\mathrm{u}}=1$, respectively. $\boldsymbol{C}^{(g)}$ represents the covariance matrix that adapts the shape of the mutation distribution to the fitness landscape of an optimization problem. The evolution paths $\boldsymbol{p}_{\sigma}^{(g)}$ and $\boldsymbol{p}_{\boldsymbol{c}}^{(g)}$ are used for step-size adaptation and covariance matrix adaptation, respectively.

Given the distribution parameters, a candidate solution $\boldsymbol{\phi}_{i}^{(g+1)}$ is generated [Fig. 2(b)]

$$
\boldsymbol{\phi}_{i}^{(g+1)}=\boldsymbol{m}^{(g)}+\boldsymbol{\sigma}^{(g)} \boldsymbol{B} \boldsymbol{D} \boldsymbol{z}_{i}^{(g+1)}
$$

where $i$ indexes the candidate solution number, $\boldsymbol{D}$ is a diagonal matrix, the elements of which are the square roots of the eigenvalues of $\boldsymbol{C}^{(g)}$, and $\boldsymbol{B}$ is an orthogonal matrix, the columns of which are the eigenvectors of $\boldsymbol{C}^{(g)}$, which are matrices given reiteratively by $\boldsymbol{C}^{(g)}=\boldsymbol{B} \boldsymbol{D}^{2} \boldsymbol{B}^{t}$.

Candidate solutions are evaluated by the finite-element analyses and are ranked based on the evaluations [Fig. 2(c)]. The difference between the mean vector and candidate solutions is obtained [Fig. 2(d)] specifically

$$
\boldsymbol{y}_{i: \lambda}^{(g+1)}=\left(\boldsymbol{\phi}_{i: \lambda}^{(g+1)}-\boldsymbol{m}^{(g)}\right) / \sigma^{(g)},
$$

where $\lambda$ represents the numbers of candidate solutions and $\boldsymbol{\phi}_{i: \lambda}^{(g+1)}$ is the $i$ th-ranked candidate solution among $\boldsymbol{\phi}_{1}^{(g)}, \ldots$, $\boldsymbol{\phi}_{\lambda}^{(g+1)}$.

The parameters of the distribution are updated using the three schemes [Figs. 2(e)-2(g)] 
(a)

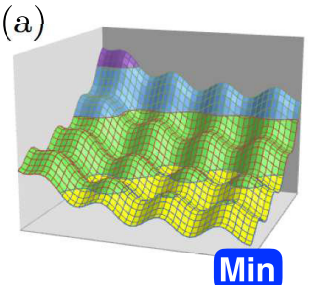

(b)
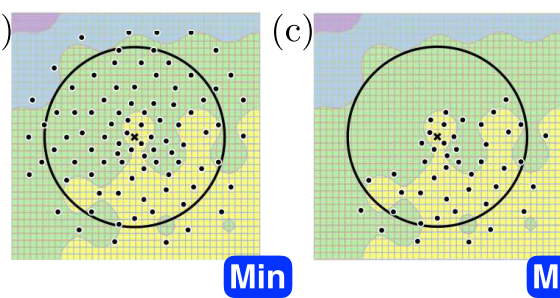

Min
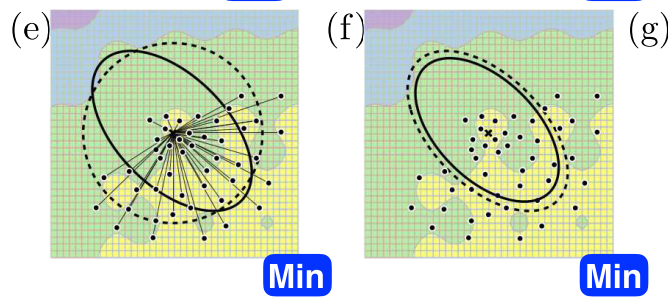

(g)

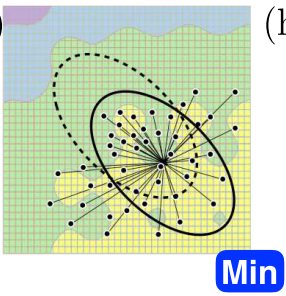

(d)

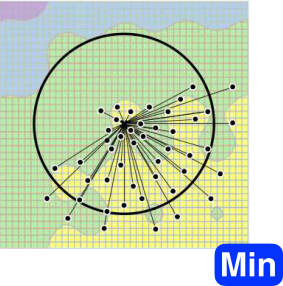

Min

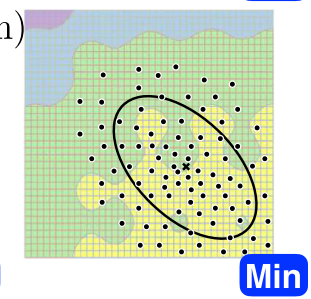

FIG. 2. Illustration of the algorithm of CMA-ES

$$
\begin{aligned}
\boldsymbol{C}^{(g+1)}= & \boldsymbol{C}^{(g)}+c_{1}\left(\boldsymbol{p}_{\boldsymbol{c}}^{(g+1)}\left(\boldsymbol{p}_{\boldsymbol{c}}^{(g+1)}\right)^{\mathrm{T}}-\boldsymbol{C}^{(g)}\right) \\
& +c_{\mu} \sum_{i=1}^{\lambda} w_{i}\left(\boldsymbol{y}_{i: \lambda}^{(g+1)}\left(\boldsymbol{y}_{i: \lambda}^{(g+1)}\right)^{\mathrm{T}}-\boldsymbol{C}^{(g)}\right), \\
\sigma^{(g+1)}= & \sigma^{(g)} \exp \left(\frac{c_{\sigma}}{d_{\sigma}}\left(\frac{\left\|\boldsymbol{p}_{\sigma}^{(g+1)}\right\|}{\mathrm{E}[\|\mathcal{N}(0, \mathbf{I})\|]}-1\right)\right), \\
\boldsymbol{m}^{(g+1)}= & \sum_{i=1}^{\mu} w_{i} \boldsymbol{\phi}_{i: \lambda}^{(g+1)}
\end{aligned}
$$

where $c_{1}$ and $c_{\mu}$ are the learning rates of rank-one and rank- $\mu$ updates for $\boldsymbol{C}$, respectively, $c_{\sigma}$ represents the learning rate for the accumulation of the step size, $d_{\sigma}$ represents the damping parameter for step-size adaptation, $\mathrm{E}\left[{ }^{*}\right]$ gives the expectation value of its argument, $\mu$ represents the numbers of higher-ranked solutions, and $w_{i}$ represents the weight assigned to $\phi_{i: \lambda}^{(g+1)}$. The evolution paths are updated using recursions

$$
\begin{aligned}
& \boldsymbol{p}_{\sigma}^{(g+1)}=\left(1-c_{\sigma}\right) \boldsymbol{p}_{\sigma}^{(g)}+\sqrt{c_{\sigma}\left(2-c_{\sigma}\right) \mu_{\mathrm{eff}}} \boldsymbol{B} \sum_{i=1}^{\mu} w_{i} z_{i: \lambda}^{(g+1)}, \\
& \boldsymbol{p}_{\boldsymbol{c}}^{(g+1)}=\left(1-c_{\boldsymbol{c}}\right) \boldsymbol{p}_{\boldsymbol{c}}^{(g)}+\sqrt{c_{\boldsymbol{c}}\left(2-c_{\boldsymbol{c}}\right) \mu_{\mathrm{eff}}} \sum_{i=1}^{\mu} w_{i} \boldsymbol{y}_{i: \lambda}^{(g+1)},
\end{aligned}
$$

where $\mu_{\text {eff }}$ represents the variance of the effective selection mass and is equal to the reciprocal of the sum of squares of $w_{i} ; c_{c}$ represents the learning rate for the accumulation for the rank-one update of $\boldsymbol{C}^{(g)}$; and $\boldsymbol{z}_{i: \lambda}^{(g+1)}$ is the random number used to produce the $i$ th-ranked solution $\boldsymbol{\phi}_{i: \lambda}^{(g+1)}$.

One of the attractive properties of the CMA-ES for practitioners is that all the parameters of the strategy such as $\lambda, \mu$, $w_{i}$, and the learning rate parameters, have well-studied default values, both theoretical and experimental, that depend solely on the dimension $N$ of the search space. We refer to Ref. 30 for default values.

The effect of a bare insulator on the temperature distribution is shown in Fig. 3. A thermal insulator $\Omega_{\text {ins }}$ makes a difference to the temperature distribution with its contour lines [Figs. 3(b) and 3(e)]. To accentuate the difference between the above two distributions, we plotted the difference in temperatures using the fine range $-0.01 \leq T^{\mathrm{n}}-T_{\text {ref }}^{\mathrm{n}} \leq 0.01$ in Figs. 3(c) and 3(f).
The results of topology optimizations based on CMAES for thermal cloaks are presented in Fig. 4. We first mention the performances of topology optimized thermal cloaks represented by the value of $\Psi$ and illustrated by the rightmost images in Fig. 4. The optimal configuration succeeds in reproducing the reference temperature distribution by minimizing the difference $T-T_{\text {ref }}$ to approximately $1 \%$ of the difference in the absence of cloaking despite the relatively tight perimeter constraint, specifically, $\tau=1 \times 10^{-2}$ [Fig. 4(a)]. The aluminum structures are placed above and below the obstacle; these structures are considered to be indispensable when they are designed subject to the relatively tight perimeter constraint. The structures above and below work to maintain the thermal conductivity in the $x$ direction reduced by the presence of the thermal insulator. Similar configurations, so-called "Eyelid" structures, are obtained through topology optimizations in previous work. . $^{14,15,18,20}$ Designed optimal configurations for wave propagations governed by Helmholtz equations ${ }^{14,15,18}$ have more complex structures with winding boundaries than those for which conduction is governed by Laplace's equation $\mathrm{s}^{20}$ because cloaks for wave propagations must control scatterings and interferences. Such eyelid structures were obtained from topology optimization with $\tau=1 \times 10^{-3}$ and were extended left and right of the obstacle as the value of $\tau$ diminishes. Under

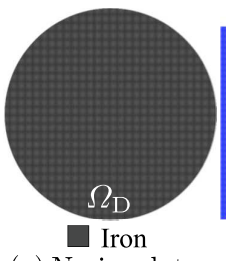

(a) No insulator

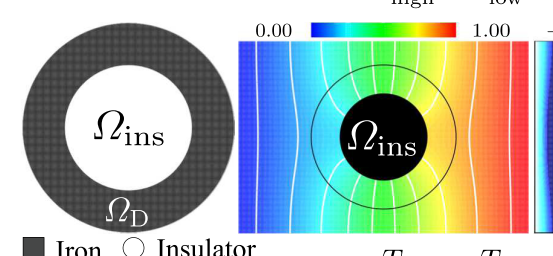

(d) No cloak

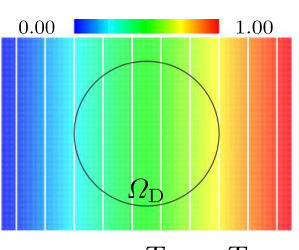

(b) $T_{\text {ref }}^{\mathrm{n}}=\frac{T_{\text {ref }}-T_{\text {low }}}{T_{\text {high }}-T_{\text {low }}}$

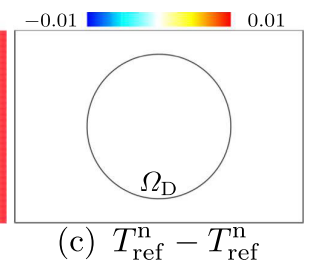

(c) $T_{\text {ref }}^{\mathrm{n}}-T_{\text {ref }}^{\mathrm{n}}$

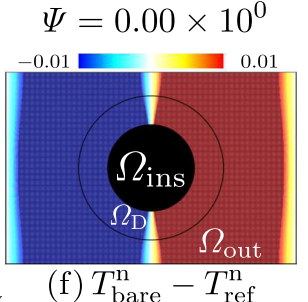

$\Psi=1.00 \times 10^{0}$
FIG. 3. (a) No insulating obstacle in $\Omega_{\mathrm{D}}$, (b) Referenced temperature, (c) $T_{\text {ref }}^{\mathrm{n}}-T_{\text {ref }}^{\mathrm{n}}(=0)$, (d) A bare obstacle, (e) Temperature around the bare obstacle, and (f) $T_{\text {bare }}^{\mathrm{n}}-T_{\text {ref }}^{\mathrm{n}}$. 


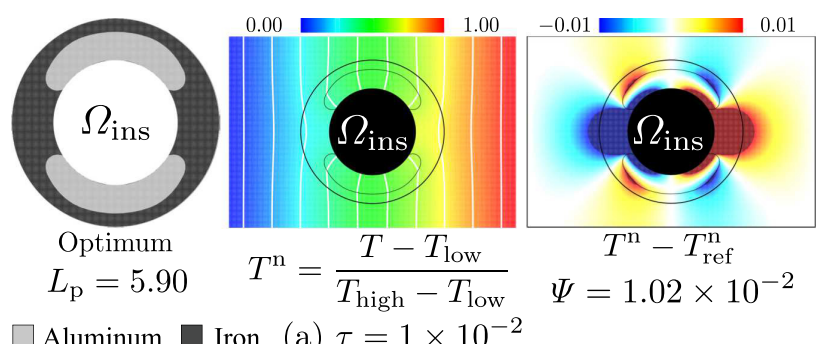

$\square$ Aluminum $\square$ Iron (a) $\tau=1 \times 10^{-2}$

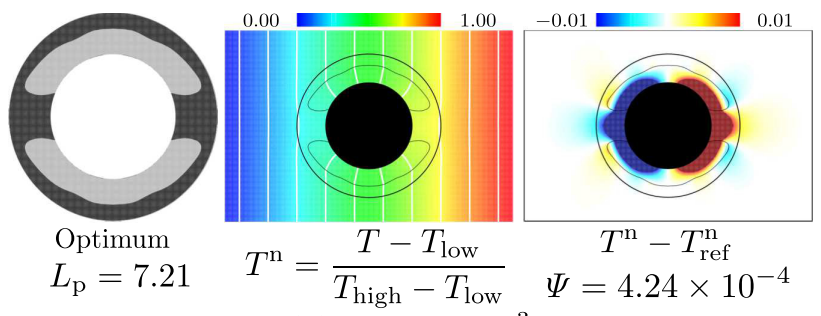

(b) $\tau=1 \times 10^{-3}$

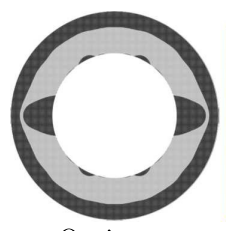

Optimum $L_{\mathrm{p}}=9.06$

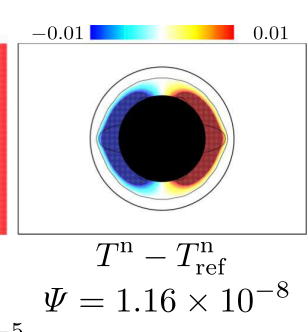

(c) $\tau=1 \times 10^{-5}$

FIG. 4. Topology-optimized thermal cloaking structures (left), cloaked temperatures (center), and difference between the cloaked temperature field and the reference field (right). The numbers of candidate solutions are set to $\lambda=140$.

relatively lax perimeter constraints, the resultant configurations exhibit a much greater degree of design freedom and optimization puts a high priority on cloaking performance. The $\Psi$-value then reaches $\Psi=1.16 \times 10^{-8}$ which is considered as nearly perfect cloaking because the difference does not show up in the rightmost image, Fig. 4(c), despite the fine range $-0.01 \leq T^{\mathrm{n}}-T_{\text {ref }}^{\mathrm{n}} \leq 0.01$. All the configurations in Fig. 4 have much simpler structures composed of iron and aluminum when compared to devices designed through transformation thermodynamics such as the seven sublayered structure made from five different ingredient sheets, ${ }^{25}$ and the bulk copper plate with numerous holes, ${ }^{24}$ and that of active thermal cloak ${ }^{31}$ composed of absorbers and emitters of heat. Besides the simplicities of structures and compositions, topology-optimized cloaks, particularly designed with $\tau=1 \times 10^{-5}$, demonstrate sufficiently comparable performances as reflected in the almost-straight contour lines in $\Omega_{\text {out }}$.

Optimal configurations and their performances obtained by topology optimization certainly depend on the properties of the object to be cloaked and the angle of heat flow because it provides optimal configurations under a given scheme. When a cloaked object is changed to a good thermal conductor instead of an insulator, the cloak is considered to consist of bad thermal conductive elements that reduce the total thermal conductivity that was increased by the cloaked object. When the angle of heat flow changes, the performances of the optimal configurations in Fig. 4 become significantly inferior. Under the heat flow along the $y$ axis, the performances of optimal configurations in Figs. 4(a)-4(c)

become $\Psi=7.95 \times 10^{-1}, 4.24 \times 10^{-1}$, and $1.67 \times 10^{-1}$, respectively.

To increase the angle of thermal invisibility, we numerically demonstrate a topology optimization implementing structural symmetries not only about the $x$ and $y$ axes but also the $y= \pm x$ axes. The results are shown in Fig. 5 . Despite the reduction in structural design freedom with the $y= \pm x$ symmetries, the $\Psi$-value is better, $\Psi=3.31 \times 10^{-9}$ [Fig. 5(c)], than that obtained by optimization without those symmetries, specifically, $\Psi=1.16 \times 10^{-8}$ [Fig. 4(c); rightmost image]. By imposing the $y= \pm x$ symmetries, the number of design variables drops from $N=4113$ to $N=2069$. This reduction is considered to promote the search for optimal solutions (configuration) using the CMA-ES. For the confirmation, the performance of the multidirectional thermal cloak in Fig. 5(a) under heat flow along the $y$ axis is evaluated as $\Psi=2.10 \times 10^{-8}$. The slight difference between the performances under the two directional heat flows is caused by the evaluation in rectangular $\Omega_{\text {out }}$. Here, we highlight the optimal configuration shown in Fig. 5(a) obtained with four axial symmetries $(x, y$, and $y= \pm x)$; the configuration seems however to be almost rotationally symmetric. The optimal configuration in Fig. 5(a) is similar to the structure of the bilayer cloak. ${ }^{28}$ By setting a smaller additional aluminum cylinder in $\Omega_{\text {ins }}$ as an alternative cloaked object, the insulator $\Omega_{\text {ins }}$ and designed aluminum $\Omega_{\mathrm{S}}$ in Fig. 5(a) are then understood to be the inner (polystyrene) and outer layers (alloy) of the bilayer cloak, respectively. Although the ratios of thermal conductivity in the outer layers to that in the host background is different, i.e., $\lambda_{\text {alum }} / \lambda_{\text {iron }}=204 /$ $67=3.04$ and $9.8 / 2.3=4.26$ in Ref. 28 , the optimization result presented well-follows those of the bilayer cloak because the widths of the bilayer cloak are also optimally chosen for determining thermal invisibility.

We confirm the performance of a completely rotational symmetric configuration obtained by $W_{\text {rot }}=W_{\text {taxes }}$ as a thermal cloak operating in an isotropic heat flux. The results are shown in Fig. 6. We note that the structural isotropy in Fig. 5 is derived from the optimization and in Fig. 6 is imposed $a$ priori. The performance of the rotational symmetric configuration reaches $\Psi=7.82 \times 10^{-6}$ under the heat flow along the $x$ axis, which is sufficiently low to operate as a thermal cloak; see Fig. 6(c), and $\Psi=9.64 \times 10^{-6}$ under the heat flow along the $y$ axis. We estimate the permissible range of fabrication for the width $W_{\text {rot }}$ based on a criterion $\Psi \leq 1.02$ $\times 10^{-2}$ obtained under $\tau=1 \times 10^{-2}$ in Fig. 4(a). The above criterion is satisfied in the range $0.214 \leq W_{\text {rot }} / R_{\mathrm{D}} \leq 0.274$,

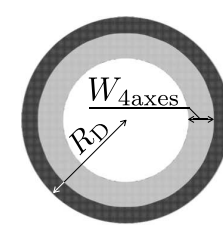

(a) $L_{\mathrm{p}}=7.29$

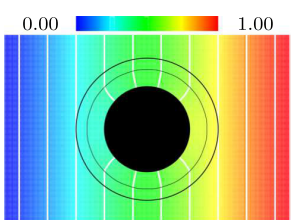

(b) $T^{\mathrm{n}}=\frac{T-T_{\text {low }}}{T_{\text {ligh }}-T_{\text {low }}}$

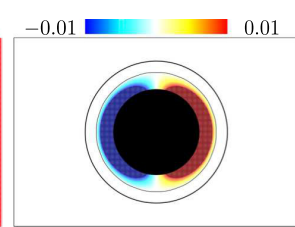

(c) $T^{\mathrm{n}}-T_{\mathrm{ref}}^{\mathrm{n}}$
FIG. 5. Results of topology optimization obtained with structural symmetries not only about the $x$ and $y$ axes but also about the $y= \pm x$ axes under $\tau=1 \times 10^{-5}$. The width of the configuration along the $x$ axis is $W_{4 a x e s}$ $=0.243 \times R_{\mathrm{D}}$. The numbers of candidate solutions are set to $\lambda=140$. 


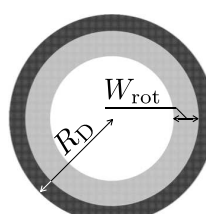

(a) $W_{\text {rot }} / R_{\mathrm{D}}$ $=0.243$

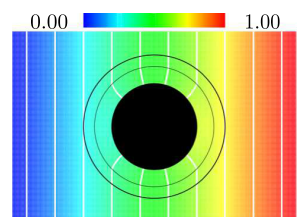

(b) $T^{\mathrm{n}}=\frac{T-T_{\text {low }}}{T_{\text {high }}-T_{\text {low }}}$

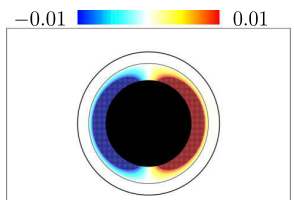

(c) $T^{\mathrm{n}}-T_{\text {ref }}^{\mathrm{n}}$ $\Psi=7.82 \times 10^{-6}$

FIG. 6. Rotationally symmetric structure obtained with $W_{\text {rot }}=W_{4 a x e s}$ $=0.243 \times R_{\mathrm{D}}$, temperature around the structure, and its performance as a thermal cloak.

and the permissible range of $W_{\text {rot }}$ is estimated as $\Delta W_{\text {rot }}$ $=(0.274-0.214) R_{\mathrm{D}}=0.1 R_{\text {ins. }}$. In the strictest experimental case, ${ }^{28} R_{\text {ins }}$ is set to $6 \mathrm{~mm}$ and the above $\Delta W_{\text {rot }}$ is estimated at $0.6 \mathrm{~mm}$.

For this study, we formulated a topology optimization for thermal cloaks based on the CMA-ES and a level-set method with an explicit boundary expression. Topology-optimized structures with various structural constraints were presented that yielded superior performances, which in some cases reached almost $0.00000116 \%$ in the absence of cloaking. By imposing additional structural symmetries, specifically about $y= \pm x$, the number of design variables was reduced and the reduction provided further improvements in the performance of the optimal configuration. Through topology optimization subject to four-axial symmetries, we obtained concept designs of thermal cloaking for isotropic heat flux. A thermally insulated object can be cloaked using a rotationally symmetric structure from isotropic inflow of heat.

This work was supported by JSPS KAKENHI (Grant No. 17K17778).

${ }^{1}$ J. B. Pendry, D. Schurig, and D. R. Smith, Science 312, 1780 (2006).

${ }^{2}$ U. Leonhardt, Science 312, 1777 (2006).

${ }^{3}$ D. Schurig, J. J. Mock, B. J. Justine, S. A. Cummer, J. B. Pendry, A. F. Starr, and D. R. Smith, Science 314, 977 (2006).

${ }^{4}$ Y. Yang, L. Jing, B. Zheng, R. Hao, W. Yin, E. Li, C. M. Soukoulis, and H. Chen, Adv. Mater. 28, 6866 (2016).
${ }^{5}$ M. Selvanayagam and G. V. Eleftheriades, Phys. Rev. X 3, 041011 (2013).

${ }^{6}$ L. Shen, B. Zheng, Z. Liu, Z. Wang, S. Lin, S. Dehdashti, E. Li, and H. Chen, Adv. Opt. Mater. 3, 1738 (2015).

${ }^{7}$ Y. Lai, H. Chen, Z.-Q. Zhang, and C. T. Chan, Phys. Rev. Lett. 102, 093901 (2009).

${ }^{8}$ S. A. Cummer and D. Schurig, New J. Phys. 9,45 (2007).

${ }^{9}$ H. Chen and C. T. Chan, Appl. Phys. Lett. 91, 183518 (2007).

${ }^{10}$ F. Yang, Z. L. Mei, T. Y. Jin, and T. J. Cui, Phys. Rev. Lett. 109, 053902 (2012).

${ }^{11}$ F. Yang, Z. L. Mei, X. Y. Yang, T. Y. Jin, and T. J. Cui, Adv. Funct. Mater. 23, 4306 (2013).

${ }^{12}$ Y. A. Urzhumov and D. R. Smith, Phys. Rev. Lett. 107, 074501 (2011).

${ }^{13}$ M. P. Bendsøe and N. Kikuchi, Comput. Methods Appl. Mech. Eng. 71, 197 (1988).

${ }^{14}$ J. Andkjær, N. A. Mortensen, and O. Sigmund, Appl. Phys. Lett. 100, 101106 (2012).

${ }^{15}$ G. Fujii, H. Watanabe, T. Yamada, T. Ueta, and M. Mizuno, Appl. Phys. Lett. 102, 251106 (2013).

${ }^{16}$ T. Yamada, H. Watanabe, G. Fujii, and T. Matsumoto, IEEE Trans. Magn. 49, 2073 (2013).

${ }^{17}$ N. Kishimoto, K. Izui, S. Nishiwaki, and T. Yamada, Appl. Phys. Lett. 110, 201104 (2017)

${ }^{18}$ G. Fujii, M. Takahashi, and Y. Akimoto, Comput. Methods Appl. Mech. Eng. (2018), https://doi.org/10.1016/j.cma.2018.01.008.

${ }^{19}$ G. Fujii and T. Ueta, Phys. Rev. E 94, 043301 (2016).

${ }^{20}$ G. Fujii, Y. Akimoto, and M. Takahashi, "Achieving direct current electric invisibility through a topology optimization based on CMA-ES," J. Appl. Phys. (submitted).

${ }^{21}$ C. Z. Fan, Y. Gao, and J. P. Huang, Appl. Phys. Lett. 92, 251907 (2008).

${ }^{22}$ T. Chen, C.-N. Weng, and J.-S. Chen, Appl. Phys. Lett. 93, 114103 (2008).

${ }^{23}$ S. Guenneau, C. Amra, and D. Veynante, Opt. Exp. 20, 8207 (2012).

${ }^{24}$ R. Schittny, M. Kadic, S. Guenneau, and M. Wegener, Phys. Rev. Lett. 110, 195901 (2013).

${ }^{25}$ Y. Ma, L. Lan, W. Jiang, F. Sun, and S. He, NPG Asia Mater. 5, e73 (2013).

${ }^{26}$ E. M. Dede, T. Nomura, and J. Lee, Struct. Multidiscip. Optim. 49, 59 (2014).

${ }^{27}$ T. Han, T. Yuan, B. Li, and C.-W. Qiu, Sci. Rep. 3, 1593 (2013).

${ }^{28}$ T. Han, X. Bai, D. Gao, J. T. L. Thong, B. Li, and C.-W. Qiu, Phys. Rev. Lett. 112, 054302 (2014).

${ }^{29}$ H. Xu, X. Shi, F. Gao, H. Sun, and B. Zhang, Phys. Rev. Lett. 112, 054301 (2014).

${ }^{30}$ N. Hansen, preprint arXiv:1604.00772 (2016).

${ }^{31}$ D. M. Nguyen, H. Xu, Y. Zhang, and B. Zhang, Appl. Phys. Lett. 107, 121901 (2015). 